\title{
Hypoglycemia Reduction and Changes in Hemoglobin A1c in the ASPIRE In-Home Study
}

\author{
Ram Weiss, MD, PhD, Satish K. Garg, MD, ${ }^{2}$ Bruce W. Bode, MD, Timothy S. Bailey, MD, \\ Andrew J. Ahmann, MD, Kenneth A. Schultz, MD, John B. Welsh, MD, PhD, \\ and John J. Shin, $\mathrm{PhD}^{6}$ for the ASPIRE In-Home Study Group
}

\begin{abstract}
Background: ASPIRE In-Home randomized 247 subjects with type 1 diabetes to sensor-augmented pump therapy with or without the Threshold Suspend (TS) feature, which interrupts insulin delivery at a preset sensor glucose value. We studied the effects of TS on nocturnal hypoglycemia $(\mathrm{NH})$ in relation to baseline hemoglobin A1c (A1C) and change in A1C during the study.

Materials and Methods: NH event rates and mean area under curve (AUC) of NH events were evaluated at different levels of baseline A1C $(<7 \%, 7-8 \%$, and $>8 \%)$ and at different levels of changes in A1C (less than $-0.3 \%$ [decreased], $-0.3 \%$ to $0.3 \%$ [stable], and $>0.3 \%$ [increased]), in the TS Group compared with the Control Group (sensor-augmented pump only).

Results: In the TS Group, $27.9 \%$ of the NH events were accompanied by a confirmatory blood glucose value, compared with 39.3\% in the Control Group. Among subjects with baseline A1C levels of $<7 \%$ or $7-8 \%$, those in the TS Group had significantly lower NH event rates than those in the Control Group $(P=0.001$ and $P=0.004$, respectively). Among subjects with decreased or stable A1C levels, those in the TS Group had significantly lower NH event rates, and the events had lower AUCs $(P \leq 0.001$ for each). Among subjects with increased A1C levels, those in the TS Group had NH events with significantly lower AUCs $(P<0.001)$.

Conclusions: Use of the TS feature was associated with decreases in the rate and severity (as measured by AUC) of NH events in many subjects, including those with low baseline A1C levels and those whose A1C values decreased during the study period. Use of the TS feature can help protect against hypoglycemia in those wishing to intensify diabetes management to achieve target glucose levels.
\end{abstract}

\section{Introduction}

$\mathbf{N}$ OCTURNAL HYPOGLYCEMIa (NH) is a major limiting factor in attaining near-normal glycemic control in patients with type 1 diabetes and is emotionally burdensome for patients and caregivers alike. ${ }^{1}$ In adults, as well as in children, the majority of severe hypoglycemic events occur during the nighttime, ${ }^{2}$ in part because of attenuation of the normal counterregulatory response observed during sleep, ${ }^{3}$ specifically during the deeper sleep phases. ${ }^{4}$ Recent analyses have shown that $\mathrm{NH}$ events are often of long duration and that low glucose levels can persist for more than $2 \mathrm{~h}$ prior to the

\footnotetext{
${ }^{1}$ Department of Human Metabolism and Nutrition and Section of Pediatric Endocrinology, Hadassah Hebrew University, Jerusalem, Israel.

${ }^{2}$ Barbara Davis Center for Diabetes, University of Colorado Denver, Aurora, Colorado.

${ }^{3}$ Atlanta Diabetes Associates, Atlanta, Georgia.

${ }_{5}^{4}$ AMCR Institute, Escondido, California.

${ }^{5}$ Oregon Health \& Sciences University, Portland, Oregon.

${ }^{6}$ Medtronic plc, Northridge, California.

The ASPIRE (Automation to Simulate Pancreatic Insulin REsponse) In-Home study is registered with ClinicalTrials.gov with clinical trial registration number NCT01497938.

(C) The Author(s) 2015; Published by Mary Ann Liebert, Inc. This Open Access article is distributed under the terms of the Creative Commons Attribution Noncommercial License (http://creativecommons.org/licenses/by-nc/4.0/) which permits any noncommercial use, distribution, and reproduction in any medium, provided the original author(s) and the source are credited.
} 
occurrence of $\mathrm{NH}$-induced seizures. ${ }^{5}$ Although nutritional strategies for $\mathrm{NH}$ prevention are routinely suggested in clinical practice, there is no evidence-based recommendation for their utility or for the optimal composition of a bedtime snack. 6

One strategy to mitigate hypoglycemia in users of sensoraugmented pump therapy is to stop insulin delivery at a predetermined sensor glucose (SG) level; the strategy is implemented as Threshold Suspend (TS) in the United States as part of the 530G system and as Low Glucose Suspend elsewhere as part of the $\mathrm{Veo}^{\mathrm{TM}}$ system (both from Medtronic plc, Northridge, CA). The strategy has been shown to reduce the duration and severity of hypoglycemic events without compromising glycemic control in an inpatient setting of exercise-induced hypoglycemia, ${ }^{7}$ in several outpatient studies, ${ }^{8-11}$ and in a retrospective analysis of anonymized data uploaded by patients as part of their routine care. ${ }^{12}$ Even in the context of elevated blood glucose levels at the time of suspension, 2-h pump suspensions appear safe and do not lead to clinically significant ketonemia. ${ }^{13}$

We wished to establish the extent of $\mathrm{NH}$ reduction associated with use of the TS feature in subjects who were stratified according to baseline hemoglobin A1c (A1C) and changes in A1C ( $\triangle \mathrm{A} 1 \mathrm{C})$ during the ASPIRE (Automation to Simulate Pancreatic Insulin REsponse) In-Home study. ${ }^{8} \mathrm{~A}$ preliminary report of these results was presented in part at the $74^{\text {th }}$ Scientific Sessions of the American Diabetes Association, held June $13-17,2014 .^{14}$

\section{Research Design and Methods}

The ASPIRE In-Home study was a multicenter, randomized, parallel study of NH-prone subjects with type 1 diabetes. ${ }^{15}$ The Control Group used sensor-augmented pump therapy, whereas the TS Group used sensor-augmented pump therapy with the addition of the TS feature. For the study, an episode of $\mathrm{NH}$ was defined as a sequence of SG values $\leq 65 \mathrm{mg} / \mathrm{dL}$ lasting $>20 \mathrm{~min}$ and starting between 10:00 p.m. and 8:00 a.m. To qualify for the study, volunteers had to have demonstrated at least two episodes of $\mathrm{NH}$ in a 2-week run-in phase. The 3-month randomized study phase was designed to provide data for between-group comparisons with respect to $\triangle \mathrm{A} 1 \mathrm{C}$ and the mean area under the curve (AUC) of $\mathrm{NH}$ events. The main results of ASPIRE In-Home included a $37.5 \%$ reduction in the mean AUC of $\mathrm{NH}$ events and a $31.8 \%$ reduction in the weekly rate of $\mathrm{NH}$ events for subjects in the TS Group compared with the Control Group, with no apparent loss in overall glycemic control determined by $\Delta \mathrm{A} 1 \mathrm{C}{ }^{8}$

In this secondary analysis of ASPIRE In-Home data, NH events were analyzed according to each subject's baseline A1C value $(<7 \%, 7-8 \%$, and $>8 \%)$ and to each subject's $\triangle \mathrm{A} 1 \mathrm{C}$ value (less than $-0.3 \%$ [decreased], $-0.3 \%$ to $0.3 \%$ [stable], and $>0.3 \%$ [increased]). Only data from the 240 subjects who provided both baseline and end-of-study A1C values were used; data from the seven subjects who withdrew early (five from the TS Group and two from the Control Group) were not used.

\section{Statistical analysis}

Secondary outcomes based on $\mathrm{NH}$ events were compared between groups, categorized by baseline A1C and $\triangle \mathrm{A} 1 \mathrm{C}$.
Event-based variables such as NH event AUC, AUC per night, nadir SG value, and duration were compared using a mixed effects model with random intercept to incorporate within-subject correlations among events. For subject-based variables, such as weekly event rate, mean SG values, mean $\mathrm{SD}$ values, and mean coefficients of variation (defined as the SD divided by the mean of a set of values) of SG values, the subject means on each variable were first calculated for each subject, and then a two-sample $t$ test was performed on the subject means data. Analyses were conducted with SAS software (version 9.3; SAS Institute, Cary, NC). All reported $P$ values are two-sided; $P<0.05$ was considered to indicate statistical significance for comparisons between groups.

\section{Results}

Of the 2,025 NH events in the TS Group, 564 (27.9\%) were accompanied by a confirmatory blood glucose value, and of the 3,002 NH events in the Control Group, 1,179 (39.3\%) were accompanied by a confirmatory blood glucose value. In addition, the NH events were verified for $41.1 \%$ (232 of 564) in the TS Group and $48.6 \%$ (573 of 1,179) in the Control Group.

\section{Effects of TS in relation to baseline A1C}

Table 1 shows that there was a significant inverse relationship between baseline A1C and NH rates in both the TS Group $(P=0.005)$ and the Control Group $(P<0.001)$, with the highest rate among subjects assigned to the Control Group who had baseline A1C values of $<7 \%$ (2.7 per subject-week) and the lowest rate among subjects assigned to the TS Group who had baseline A1C values of $>8 \%$ (1.1 per subject-week). The rate was significantly lower in the TS Group than in the Control Group for subjects with baseline A1C values of $<7 \%$ $(P=0.001)$ or $7-8 \%(P=0.004)$. The differences in rate were consistent throughout the study, as shown in Supplementary Figure S1 (Supplementary Data are available online at www.liebertonline.com/dia). Regardless of baseline A1C, $\mathrm{NH}$ events experienced by subjects in the TS Group had significantly lower AUCs and shorter durations than those experienced by subjects in the Control Group (Table 1). There was no difference between mean SG and SD between the groups. The distribution of AUC values for hypoglycemic events is given in Supplementary Figure S2.

\section{Effects of $T S$ in relation to $\triangle A 1 C$}

Table 2 shows that the between-group differences for AUC were statistically significant in all of the $\triangle \mathrm{A} 1 \mathrm{C}$ categories $(P<0.001$ for each). The percentage difference was largest for subjects with $\mathrm{A} 1 \mathrm{C}$ values that increased over the course of the study, in which the mean AUC was $46.3 \%$ lower for subjects in the TS Group compared with subjects in the Control Group. The between-group differences in $\mathrm{NH}$ rate were significant for subjects with $\mathrm{A} 1 \mathrm{C}$ values that decreased or were stable over the course of the study; for those with a decrease in $\mathrm{A} 1 \mathrm{C}$, the mean rate was $37.5 \%$ lower for subjects in the TS Group compared with subjects in the Control Group (1.5 vs. 2.4 events per subject-week, respectively; $P<0.001)$. In the Control Group, subjects with stable A1C values experienced $\mathrm{NH}$ events at a rate that was lower than Control Group subjects with decreased A1C values and higher than 

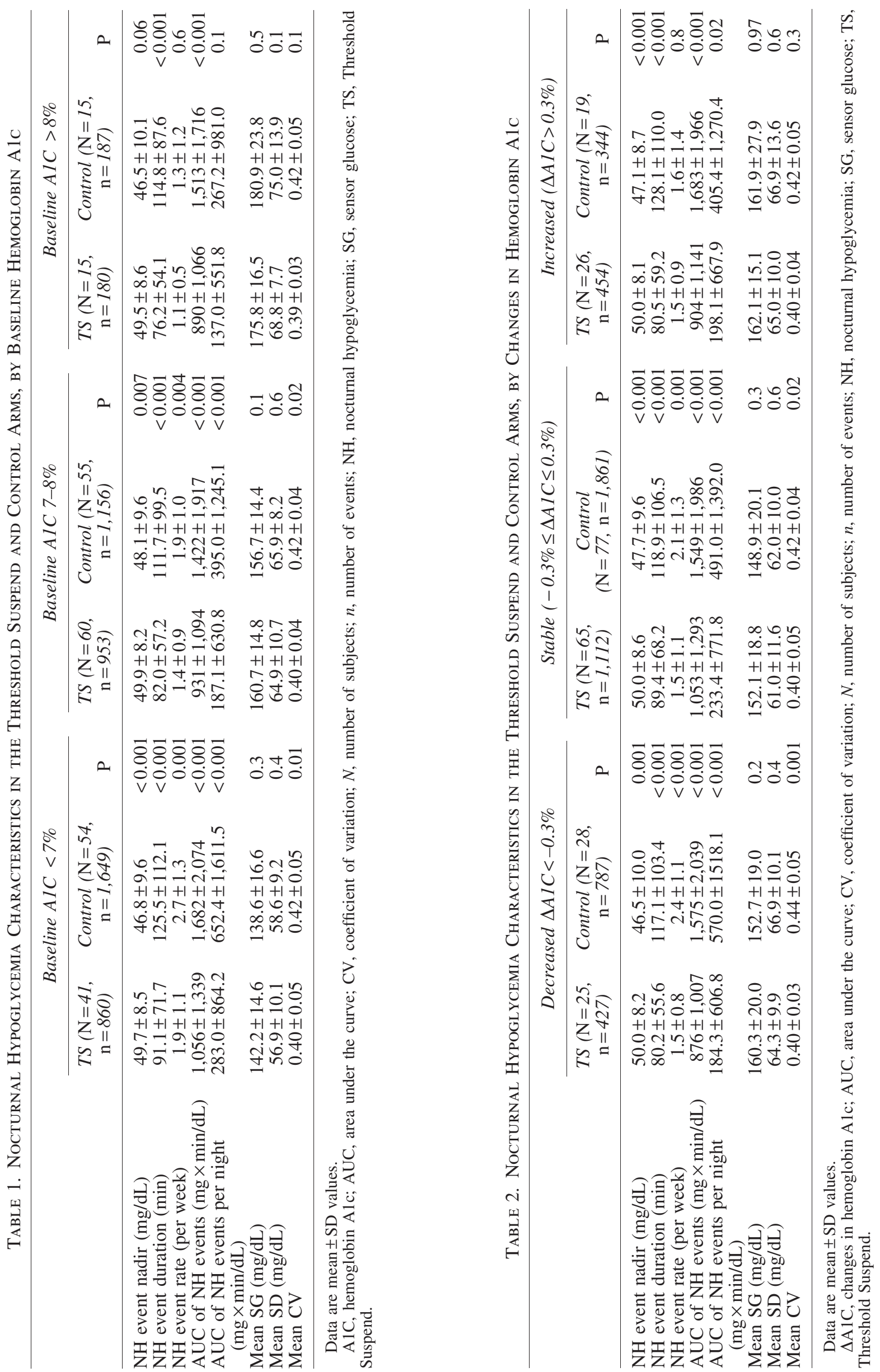
Control Group subjects with increased A1C values. In the TS Group, the NH rate averaged 1.5 per subject-week, regardless of $\triangle \mathrm{A} 1 \mathrm{C}$ category. The mean $\mathrm{NH}$ event duration was significantly shorter for subjects in the TS Group than for subjects in the Control Group, regardless of $\triangle \mathrm{A} 1 \mathrm{C}$ category $(P<0.001$ for each between-group comparison), as was the nadir SG. Among subjects with decreased or stable A1C values, glycemic variability (measured as coefficient of variation) was significantly lower among subjects in the TS
Group than among subjects in the Control Group, although mean SG and SD were not different.

\section{Effects of TS in relation to both baseline $A 1 c$ and $\triangle A 1 C$}

Figure 1 shows NH event rates and AUCs among subjects stratified by both baseline and $\triangle \mathrm{A} 1 \mathrm{C}$ values. Reductions in $\mathrm{NH}$ event rate attributable to TS Group assignment were seen for subjects with baseline A1C of $<7 \%$ who maintained
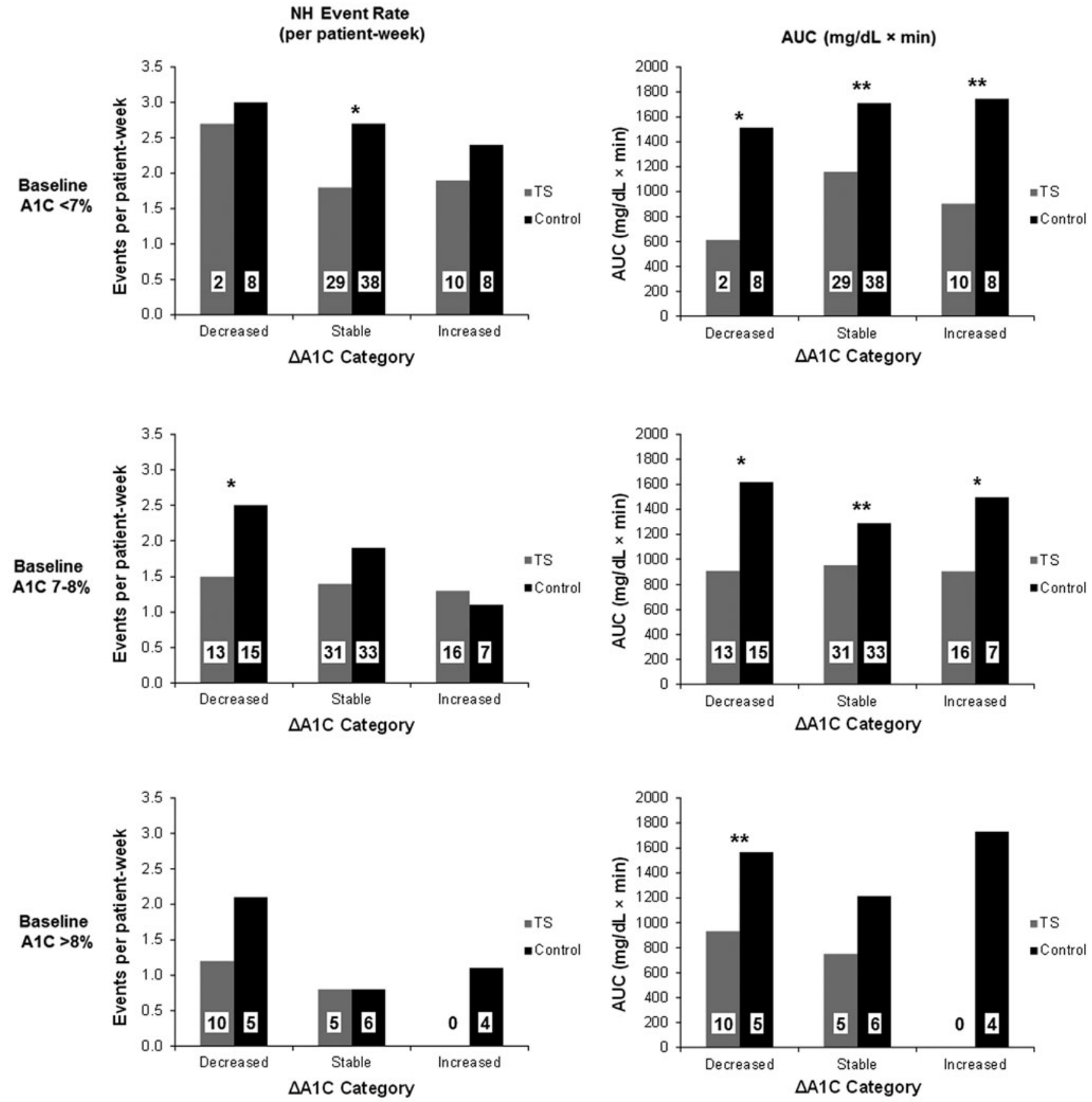

FIG. 1. (Left panels) Nocturnal hypoglycemia (NH) event rate and (right panels) area under the curve (AUC) of NH events for subjects stratified according to baseline hemoglobin A1c (A1C) $(<7 \%, 7-8 \%$, or $>8 \%$ in the top, middle, and bottom panels, respectively), changes in $\mathrm{A} 1 \mathrm{C}(\triangle \mathrm{A} 1 \mathrm{C})$ category (decreased, stable, or increased in the left, middle, and right pairs of columns in each panel), and treatment group assignment (Threshold Suspend [TS] [gray columns] or Control [black columns]). The number of subjects is shown in each column. $* P<0.05$, $* * P<0.005$ for between-treatment group comparisons. 
stable A1C values and for subjects with baseline A1C of $7-8 \%$ whose A1C values decreased during the study. For subjects with baseline A1C of $<7 \%$ or with baseline A1C of $7-8 \%$, significant reductions in the AUC of $\mathrm{NH}$ events were seen among subjects assigned to the TS Group, regardless of $\triangle \mathrm{A} 1 \mathrm{C}$ category.

\section{Discussion}

The TS feature was designed to reduce the severity and duration of hypoglycemia by suspending insulin delivery upon detection of a predefined low SG value. In the ASPIRE In-Home study, the initial threshold for suspension of insulin was set to $70 \mathrm{mg} / \mathrm{dL}$, and this value was maintained for $97.6 \%$ of the subject-hours of the study. Evidence from earlier observational $^{7,9,10}$ and randomized controlled ${ }^{8,11}$ studies suggested that the TS feature significantly reduces the rate and severity of hypoglycemia as measured by SG values.

The Control Group appears to have a higher patient reaction rate to $\mathrm{NH}$ events. It is likely that the event rate in the TS Group was inflated more than in the Control Group, yet it was observed that the event rate in the TS Group was lower than that in the Control Group.

$\mathrm{NH}$ remains a critical barrier that limits treatment intensification in type 1 diabetes mellitus. ${ }^{16}$ Many patients with type 1 diabetes mellitus experience $\mathrm{NH}$ frequently, with many events lasting longer than $120 \mathrm{~min} .{ }^{17}$ The ASPIRE In-Home Study showed that use of the TS feature reduced the mean and median duration of $\mathrm{NH}$ events by 39.4 and $11.9 \mathrm{~min}$, respectively, ${ }^{8}$ to values that are less than the reported time typically associated with the development of seizures. ${ }^{5}$ These effects were mostly independent of the degree of metabolic control such as baseline $\mathrm{A} 1 \mathrm{C}$ or $\triangle \mathrm{A} 1 \mathrm{C}$ from study beginning to end. In addition, no subjects in the TS Group had severe hypoglycemia, whereas four subjects in the Control Group had severe hypoglycemia during the 3 -month study time period. ${ }^{8}$

The effect of the regular use of the TS feature in subjects who lowered their A1C values during the course of the study is of interest, given the association between lower A1C values and higher rates of hypoglycemia. ${ }^{18}$ Sensor-augmented pumps have been shown to allow A1C reduction without a significant effect on hypoglycemia rates. ${ }^{19}$ Despite the fact that the aim of the ASPIRE In-Home study was to test safety and not to reduce $\mathrm{A} 1 \mathrm{C}$, there was a subgroup of patients who did, and many patients with initially low A1C values $(<7 \%)$ were able to sustain this value throughout the study. Subjects who maintain or achieve excellent glycemic control can benefit through routine use of the TS feature.

The study had several limitations. First, SG values were used for all analyses without validation by a reference method. Second, because blood glucose values were not obtained during many of the $\mathrm{NH}$ events and because the timing and extent of carbohydrate intake were often not recorded, our ability to determine sensor accuracy around the time of $\mathrm{NH}$ events is limited. However, sensor accuracy is unlikely to depend on treatment group assignment and unlikely to have biased our conclusions. Third, the generalizability of the study results may be limited because only hypoglycemia-prone subjects were enrolled. The results may not be generalizable to sensors with different performance characteristics, and the reported rates of hypoglycemia are likely to be inflated (in both groups). Finally, the 3-month duration of the study may have been insufficient to demonstrate long-term benefits of the TS feature.

This analysis demonstrates the protective effects of the TS feature above and beyond $\mathrm{NH}$ prevention. NH occurred at lower rates among subjects in the TS Group throughout the study. When NH events did occur in the TS Group, they tended to be shorter and characterized by higher nadir SG values than events in the Control Group. Additional strategies will probably be needed to reduce the severity and rate of $\mathrm{NH}$ even further. This modality is the first automated insulin delivery module in practice and may pave the way for the ultimate goal-the closed-loop artificial pancreas. ${ }^{20}$

The current analysis of ASPIRE In-Home data showed that overall rates, durations, and severities of $\mathrm{NH}$ events were lower among subjects who regularly used the TS feature than among those who did not. The benefits were mostly independent of baseline $\mathrm{A} 1 \mathrm{C}$ levels and $\Delta \mathrm{A} 1 \mathrm{C}$ levels. In addition, this analysis showed that for those with an baseline A1C level of $<7 \%$ who maintained glucose control and for those with a baseline A1C level of 7-8\% who reduced their A1C during the 3-month study period, the TS feature allowed improving metabolic control safely by reducing rates of $\mathrm{NH}$ and AUC of hypoglycemic events.

\section{Acknowledgments}

The ASPIRE In-Home Study was funded by Medtronic, plc.

\section{Author Disclosure Statement}

R.W. is a consultant for Medtronic. J.B.W., K.A.S., and J.J.S. are employees of Medtronic, plc. Atlanta Diabetes Associates, the employer of B.W.B., has received research support from Medtronic. T.S.B. and A.J.A. have received research support from Medtronic, plc. S.K.G. has received speaking/advisory board consulting fees from Eisai, Medtronic, Novo Nordisk, Roche, the Jaeb Center, the National Institute of Diabetes and Digestive and Kidney Diseases, the JDRF, and Sanofi and research grants from Eli Lilly, Halozyme, Novo Nordisk, Merck, MannKind, Medtronic, the T1D Exchange, the National Institute of Diabetes and Digestive and Kidney Diseases, the JDRF, and Sanofi.

\section{References}

1. Haugstvedt A, Wentzel-Larsen T, Rokne B, et al.: Perceived family burden and emotional distress: similarities and differences between mothers and fathers of children with type 1 diabetes in a population-based study. Pediatr Diabetes 2011;12:107-114.

2. The Diabetes Control and Complications Trial Research Group: Epidemiology of severe hypoglycemia in the Diabetes Control and Complications Trial. Am J Med 1991;90:450-459.

3. Diabetes Research in Children Network Study Group: Impaired overnight counterregulatory hormone responses to spontaneous hypoglycemia in children with type 1 diabetes. Pediatr Diabetes 2007;8:199-205.

4. Jauch-Chara K, Hallschmid M, Gais S, et al.: Awakening and counterregulatory response to hypoglycemia during early and late sleep. Diabetes 2007;56:1938-1942.

5. Buckingham B, Wilson DM, Lecher T, et al.: Duration of nocturnal hypoglycemia before seizures. Diabetes Care 2008;31:2110-2112. 
6. Desjardins K, Brazeau AS, Strychar I, et al.: Are bedtime nutritional strategies effective in preventing nocturnal hypoglycaemia in patients with type 1 diabetes? Diabetes Obes Metab 2014;16:577-587.

7. Garg S, Brazg RL, Bailey TS, et al.: Reduction in duration of hypoglycemia by automatic suspension of insulin delivery: the in-clinic ASPIRE study. Diabetes Technol Ther 2012;14:205-209.

8. Bergenstal RM, Klonoff DC, Garg SK, et al.: Thresholdbased insulin-pump interruption for reduction of hypoglycemia. N Engl J Med 2013;369:224-232.

9. Choudhary P, Shin J, Wang Y, et al.: Insulin pump therapy with automated insulin suspension in response to hypoglycemia: reduction in nocturnal hypoglycemia in those at greatest risk. Diabetes Care 2011;34:20232025.

10. Danne T, Kordonouri O, Holder M, et al.: Prevention of hypoglycemia by using low glucose suspend function in sensor-augmented pump therapy. Diabetes Technol Ther 2011;13:1129-1134.

11. Ly TT, Nicholas JA, Retterath A, et al.: Effect of sensoraugmented insulin pump therapy and automated insulin suspension vs standard insulin pump therapy on hypoglycemia in patients with type 1 diabetes: a randomized clinical trial. JAMA 2013;310:1240-1247.

12. Agrawal P, Welsh JB, Kannard B, et al.: Usage and effectiveness of the low glucose suspend feature of the Medtronic Paradigm Veo insulin pump. J Diabetes Sci Technol 2011;5:1137-1141.

13. Sherr JL, Palau Collazo M, Cengiz E, et al.: Safety of nighttime 2-hour suspension of basal insulin in pumptreated type 1 diabetes even in the absence of low glucose. Diabetes Care 2014;37:773-779.
14. Bailey TS, Weiss R, Bode BW, et al.: Hypoglycemia reduction and changes in A1C in the ASPIRE In-Home Study [abstract 231-OR]. Diabetes 2014;63(Suppl 1):A60.

15. Klonoff DC, Bergenstal RM, Garg SK, et al.: ASPIRE InHome: rationale, design, and methods of a study to evaluate the safety and efficacy of automatic insulin suspension for nocturnal hypoglycemia. J Diabetes Sci Technol 2013;7:1005-1010.

16. Cryer PE: The barrier of hypoglycemia in diabetes. Diabetes 2008;57:3169-3176.

17. Juvenile Diabetes Research Foundation Continuous Glucose Monitoring Study Group: Prolonged nocturnal hypoglycemia is common during 12 months of continuous glucose monitoring in children and adults with type 1 diabetes. Diabetes Care 2010;33:1004-1008.

18. The Diabetes Control and Complications Trial Research Group: Hypoglycemia in the Diabetes Control and Complications Trial. Diabetes 1997;46:271-286.

19. Yeh HC, Brown TT, Maruthur N, et al.: Comparative effectiveness and safety of methods of insulin delivery and glucose monitoring for diabetes mellitus: a systematic review and meta-analysis. Ann Intern Med 2012;157:336-347.

20. Keenan DB, Mastrototaro JJ, Voskanyan G, et al.: Delays in minimally invasive continuous glucose monitoring devices: a review of current technology. J Diabetes Sci Technol 2009;3:1207-1214.

Address correspondence to: John J. Shin, PhD Medtronic, plc. 18000 Devonshire Street Northridge, CA 91325

E-mail: john.shin@medtronic.com 